\title{
Specialized pro-resolving lipid mediators (SPMs) and their potential contribution to the resolution of inflammatory processes associated to PCOS
}

Pedro-Antonio Regidor ${ }^{1}$, Anna Mueller ${ }^{2}$, Manuela Sailer ${ }^{2}$, Fernando Gonzalez Santos ${ }^{3}$, Jose Miguel Rizo ${ }^{4}$, and Fernando Moreno Egea ${ }^{3}$

${ }^{1}$ Exeltis Germany

${ }^{2}$ Exeltis Healthcare

${ }^{3}$ Solutex Soluciones Extractivas Alimentarias

${ }^{4}$ Chemo Group

October 23, 2020

\footnotetext{
Abstract

PCOS represents a chronic disease affecting between $8-10 \%$ of women during their reproductive life. Concepts were developed that re-define inflammation as being composed of the inflammatory response and active resolution processes, which occur at the same time. Key players are the specialized pro-resolving lipid mediators (SPMs).

Title: Specialized pro-resolving lipid mediators (SPMs) and their potential contribution to the resolution of inflammatory processes associated to PCOS.

Authors: Pedro-Antonio Regidor ${ }^{1,7}$, Anna Müller ${ }^{2}$, Manuela Sailer ${ }^{3}$, Fernando Gonzalez Santos ${ }^{4}$, Jose Miguel Rizo ${ }^{5}$, Fernando Moreno Egea ${ }^{6}$

Affilaitions:

${ }^{1}$ Exeltis Healthcare. Adalperostr. 84, 85737 Ismaning, Germany. Phone: +49 894520529 - 19, Mobil:

+491738938132, Fax: +49 894520529 - 819, E-Mail: pedro-antonio.regidor@exeltis.com

${ }^{2}$ Exeltis Healthcare. Adalperostr. 84, 85737 Ismaning, Germany. Phone: +49 894520529 - 19, Mobil:

+491738938145, Fax: +49 894520529 - 819, E-Mail: anna.mueller@exeltis.com

3 Exeltis Helthcare. Adalperostr. 84, 85737 Ismaning, Germany. Phone: +49 894520529 - 19, Mobil:

+491734166276, Fax: +49 894520529 - 819, E-Mail: manuela.sailer@exeltis.com

${ }^{4}$ Solutex SA. Avenida de la Transición Espanola 24, 28108 Alcobendas. Spain. Phone: +34 918060477. Mobil: +34 647775178, Fax: +34 91060605, E-Mail: fgsantos@solutexcorp.com

${ }^{5}$ OTC Chemo. Manuel Pombo Angulo 28- 4th floor, 28050 Madrid. Spain. Phone: +34 913021560 9507, Mobil: + 34 618721034. E-Mail:

josemiguel.rizo@chemogroup.com

${ }^{6}$ Solutex SA. Avenida de la Transición Espanola 24, 28108 Alcobendas. Spain. Phone: +34 918060477.

Mobil: +34 64, Fax: +34 91060605, E-Mail: fmoreno@solutexcorp.com

${ }^{7}$ responsible for correspondence
} 
Keywords: PCOS, obesity, inflammation, specialized pro-resolving mediators (SPMs)

Key Clinical Message : SPMs, which derive from 18-HEPE, 17-HDHA and 14-HDHA, and are biosynthesized from their respective precursor omega-3-fatty acids EPA and DHA, have a possible influenece on the resolution of inflammation associated with polycystic ovary snydrome.

Abstract:

PCOS represents a chronic disease affecting between $8-10 \%$ of women during their reproductive life. Concepts were developed that re-define inflammation as being composed of the inflammatory response and active resolution processes, which occur at the same time. Key players are the specialized pro-resolving lipid mediators (SPMs).

Introduction

The Polycystic Ovary Syndrome (PCOS) is a disease that causes irregular bleeding, chronic anovulation, androgen excess, and a typical ovarian ultrasound feature [1]. It affects between 5 and $10 \%$ of women in their reproductive age, thus representing one of the most frequent causes of infertility [2]. The reasons for the development of a PCOS have not been resolved yet. Genetic predisposition, together with the gestational environment and lifestyle factors seem to be key contributors. The European Society of Human Reproduction and Embryology and the American Society for Reproductive Medicine defined the criteria for the definition of this disease $[3,4]$ at a meeting in Rotterdam in 2003.

Apart from the symptoms mentioned above, $30-40 \%$ of women with PCOS show a reduced glucose tolerance [5], often accompanied by insulin resistance, which both are independent of body mass index (BMI). 80\% of the obese women and 30-40\% of the lean individuals with PCOS suffer from hyperinsulinemia [6,7]. Hence, these aspects have been addressed by several studies to reach a general agreement on diagnostic criteria. It has been found that hyperinsulinemia is a key factor in the clinical pathogenesis of PCOS with its characteristic symptoms such as hyperandrogenism, chronic anovulation, typical PCOS ultrasound images, and skin issues such as acne, hirsutism, and seborrhea and seems to be independent of weight [8]. Excess insulin may lead to enhanced androgen synthesis by direct stimulation of the androgen production on the one hand and by reducing the serum levels of sex hormone-binding globulin (SHBG) on the other side [9].

However, the manifestation of PCOS is heterogeneous, and its etiopathology is still unclear [10,11]. As proven by familial clustering of PCOS incidence, results from twin studies, and the fact that specific endocrine and metabolic features of PCOS are heritable, genetic factors play an important role in the pathogenesis [10, $11,12]$. It was further shown that embryos, which are exposed to unusually high levels of androgens and anti-Müller-hormone (AMH), have an increased risk for developing a PCOS later in life [13]. Additional factors, such as obesity, further have a substantial impact on the severity of PCO symptoms [14, 15].

Obesity and inflammation

A state of chronic systemic inflammation is characteristic of obesity and can be determined by measuring increased serum levels of inflammatory cytokines and altered frequencies and functions of peripheral blood lymphocytes $[16,17,18]$. These changes are manifested at the tissue level and in adipose-, liver- and other tissue beds $[17,18]$. They might be responsible for comorbidities that are often related to obesity, such as atherosclerosis, diabetes, and steatohepatitis [19, 20, 21, 22, 23]. This kind of inflammation is often attributed to irregularities in innate immunity. However, innate and adaptive immune systems are closely interlinked, and consequently, obesity-related inflammation is associated with both processes [24].

Inflammatory processes occur due to traumatic events or infections and are also crucial for the turnover of cells during aging [25]. In this context, it is involved in the regulation of essential processes associated with cellular homeostasis, such as proliferation, necrosis, and apoptosis. Consequently, also by-products of cell necrosis and apoptosis, i.e., endogenous stimuli, can trigger inflammatory responses that are necessary for the regulation of tissue turnover. In obese individuals, systemic levels of free fatty acids are elevated, for example. These molecules are primary ligands of Toll-like receptors, which themselves are critical regulators 
of the innate immune response $[26,27]$. In this way, the systems, which regulate obesity and inflammation, are linked directly.

From a molecular point of view, there is significant crosstalk between the intracellular signalling pathways of inflammation and glucose homeostasis, since they have multiple signalling mediators in common.

A further relationship between inflammation and the metabolic system is visible on the cellular level, since adipocytes and macrophages are closely related, and their evolution might be traced back to a conventional primordial precursor cell [28].

It has also been demonstrated that insulin resistance and inflammatory processes are closely linked and may stimulate each other [29]. Both, subclinical inflammation and insulin resistance are essential makers for the development of cardiovascular disease [30] and for women with PCOS, whose cardiovascular risks are elevated, a connection between inflammation and their hormonal-metabolic features were also shown [31].

If obesity and inflammation are correlated, it is worthy of looking at the possible pathways of inflammation that accompany or influence the obesity in PCOS women considering the modern perception of inflammatory processes.

The role of inflammation

In many chronic diseases, including vascular and neurological disorders, as well as metabolic syndrome, excessive inflammatory processes are manifested, thus representing a public health concern. If the endogenous control points within the inflammatory pathways were understood completely, the pathogenesis of the diseases might become more explicit, and new approaches for treatment might be found.

When a host experiences a trauma, barrier breakage, or microbial invasion, potential invaders must be eliminated, the location must be cleared, and affected tissue must be remodelled and regenerated. For the acute inflammatory response, several lipid mediators are crucial. They include eicosanoids (prostaglandins and leukotrienes), which derive from arachidonic acid, an essential fatty acid [32, 33], and different cytokines and chemokines $[35,35,36]$. These molecules interact with each other, thereby further intensifying the inflammatory process that may, in turn, be counteracted with pharmacological inhibitors and receptor antagonists. Since inflammatory processes are involved in many prevalent diseases, it is necessary to broaden the knowledge of all mechanisms involved in order to improve the therapeutic options.

Historically, the inflammatory response used to be separated into an active initiation and a passive resolution process [37]. Recently, however, mediators were identified which have pro-resolving capacities and can be synthesized from omega-3 (n-3) essential fatty acids (EFA). Studies have shown that the resolution process can be "switched on" in animal models and may thus rather be an active response in the self-limitation of acute inflammation than a passive dilution of chemo-attractants $[38,39]$.

Molecules, which are supposed to act as mediators, must be supplied in enough amounts in order to lead to reactions in vivo. For EPA and DHA, anti-inflammatory properties have been proposed for many years. These omega-3 fatty acids compete with arachidonic acid in reducing pro-inflammatory eicosanoids [40]. However, the underlying molecular mechanisms had remained obscure until recent results emerged, and whether EPA or DHA is more relevant for human health or therapeutic options is still under debate [40].

It has been shown for resolving inflammatory exudates that omega-3 fatty acids serve as substrates for the synthesis of specific signalling molecules - the so-called specialized pro-resolving mediators (SPMs), which comprise resolvins, protectins, lipoxins and maresins (Fig. 1). These findings triggered new studies concerning the resolution pathways and the immune mechanisms underlying homeostasis. It was shown in animal models that SPMs promote critical paths of the inflammatory resolution, as they limit the infiltration of polymorphonuclear neutrophils and the elimination of apoptotic cells by macrophages [41]. (see figure 1).

Active resolution of inflammation 
Inflammations may be resolved entirely or become a chronic state. Formerly, resolution of active inflammation has been considered a passive event, upon which inflammatory mediators such as prostaglandins or cytokines were merely diluted, thus disappearing from the site of inflammation. This would finally lead to prevent leukocyte infiltration into the tissue. However, Serhan et al. provided new evidence to revise this theory by demonstrating the existence of an active resolution process mediated by so-called selective proresolving mediators (SPMs) in several studies. The SPM molecular superfamily contains subgroups named resolvins (Rvs), protectins, maresins, and lipoxins. The biosynthesis of the SPMs (with lipoxygenases and cyclooxygenases intervening both on the pathways of eicosanoids and SPMs), as well as the corresponding cell membrane receptors, have been described. SPMs are crucial for sufficient resolution of inflammatory processes, and based on these new findings, Serhan et al. described three novel pathways for the potential development of acute inflammation. They include the action of the SPMs, as well as crucial endogenous control mechanisms and, are illustrated in Fig. 2.

Importantly, within this new perception of inflammatory processes, the resolution is an active mechanism, which does not start with a delay, but at experimental timepoint Zero.

Alfa signals Omega throughout the course of inflammation, mainly SPMs were found to repress inflammatory signals by ending tissue infiltration of neutrophils and preventing further recruitment of immune cells to the site of inflammation. Subsequently, phagocytic macrophages are stimulated, which further leads to increased clearance and elimination of apoptotic polymorphonuclear neutrophils (PMNs) by efferocytosis and phagocytosis [35].

SPMs are synthesized from eicosapentaenoic acid (EPA, C20:5n-3) and docosahexaenoic acid (DHA, C22:63 ). Both are omega-three polyunsaturated fatty acids (PUFAs) and serve as precursors in the biochemical pathways leading to SPMs via the metabolites 18-HEPE, 17-HDHA, or 14-HDHA (see Fig. 3) [44].

Since the development of this new concept of inflammation, many tissues have been suitable targets for treating inflammation with SPMs or their active precursors 18-HEPE, 17-HDHA and the 14-HDHA, in order to elicit dynamic resolution of inflammation. In contrast to traditionally applied anti-inflammatory therapies, they are do not act as immunosuppressors, and debris is cleared, thus being potentially useful for the treatment of chronic inflammation. Substances, which are applied nowadays, have distinct disadvantages: steroids may interfere with wound healing, can promote osteoporosis, and is immunosuppressive. NSAIDs may lead to stomach bleeding, are potentially toxic for the cardiovascular system and the kidneys and interfere with wound healing. Cyclooxygenase (COX)2 inhibitors constitute a risk factor for cardiovascular and thromboembolic events. Anti-TNF therapies for blocking cytokines lead to increased rates of infections and enhance the risk of lymphoma development.

SPMS, on the other hand, was shown to increase the killing of microbial invaders and their clearance by immunocytes. It was demonstrated that they down-regulate infiltration and recruitment of PMN, enhance phagocytosis, and efferocytosis (M1 to M2). Application of SPMs also decreased the level of pro-inflammatory chemical mediators, while increasing the number of anti-inflammatory mediators like IL-10, for example. Finally, they can reduce inflammatory pain, stimulate the regeneration of inflamed tissue, and promote wound healing (see figure 4).

Obesity and inflammation

In obesity, adipose tissue is characterized by high levels of pro-inflammatory eicosanoids and depleted levels of SPMs. DHA EPA is required, but enzymes are critical to the formation of the SPMs circuit. SPMs increase M2 polarization of adipose tissue macrophages. In some inflammatory conditions, lack of SPMs has been related to the inability of immune cells for processing SPM substrate (EPA /DHA); thus, supplementation with EPA and DHA would be ineffective in restoring SPMs level. In those cases, supplementation with 17HDHA and 18HEPE (advanced SPMs intermediates also derived from DHA and EPA) could be effective in restoring SPMs levels [45] (see figure 5).

Administration of 17HDHA and other SPMs could be more efficient in overriding impaired formation of 
SPMs in conditions characterized by dysfunctional LOX activity. This disfunction are known and described in figure 6 .

Conclusions:

Taking into consideration that PCOS has no cure [46] and that treatment should involve lifestyle changes such as weight loss and exercise $[47,48]$ the birth control pills may help with improving the regularity of periods, excess hair growth, and acne. Metformin and anti-androgens may also help. Other typical acne treatments and hair removal techniques may be used [46].

Inositols have also been used for the improvement of the disease, and efforts to improve fertility include weight loss, clomiphene, or metformin and In vitro fertilization.

All these preventive actions are on the other site, only symptomatic treatments.

Pro-resolving mediators derived from of omega-3 fatty acids

The anti-inflammatory process is not synonymous with the pro-resolution process, in which SPMs as agonists stop the immigration of neutrophils and activate non-phlogistic macrophage reactions and resolution programs.

This difference became clear when new families of anotacoids and their descendants were identified, which can be activated by aspirin. This provided evidence in animal models that the resolution process is actively controlled by lipid mediators. Investigating and using this mechanism is an important challenge for the future as omega-3 fatty acids are currently widely used as dietary supplements but are prescribed by medical providers in less than $25 \%$ of cases. This is due to the fact, that their therapeutic benefits are assessed differently in clinical trials. This reinforces the importance to understand the mechanisms underlying their effect.

It was only by using a model system with resolving mouse exudates that the metabolic pathways and effects of the SPMs could be identified. Since then, each resolvin ( $\mathrm{RvE1}$, RvD1, RvD2, RvD3 and RvD5), protectines and maresine synthesized by leukocytes could be compared with completely organo-chemically synthesized molecules and described stereochemically [41, 49].

Models of chronic inflammatory diseases

Periodontitis is a chronic inflammatory disease in which infection leads to a tissue injury around the tooth that is neutrophil-mediated. Activated neutrophils produce PGE2, LTB4 and LXA4 in patients with periodontitis. PGE2 leads to bone loss in this tissue. P. gingivalis causes the recruitment of neutrophils in Air Pouch mouse models as well as a high regulation of COX-2. Stable LXA4 analogues reduced both neutrophil inflow and COX-2 expression associated with the oral pathogen. In addition, P. gingivalis increased the expression of COX-2 in the lungs and heart of mice. Also, ribosomale 16S RNA of P. gingivalis was present in these tissues, which provided evidence of a role of this oral pathogen in the development of systemic inflammation. Transgenic rabbits that overexpress human lipoxygenase type I produce 6 to 10 times as much LXA4 as wild rabbits [50]. These transgenic rabbits show lower bone loss in periodontitis, significantly reduced recruitment of neutrophils, and vascular permeability of the skin when compared to wild rabbits when stimulating the immune system. This suggests that overexpression of lipoxin biosynthesis has a protective effect and this finding could be useful in controlling inflammation-dependent bone degradation.

Unexpectedly, the overexpression of 15-lipoxygenase in these transgenic rabbits drastically reduces the onset of atherosclerotic lesions. In transgenic 12/15 lipoxygenase mice, RvD1, PD1 and 17-HDHA with reduced PGE2 were identified in activated macrophages (LC-MS-MS analysis [51]. LXA4, PD1 and RvD1 reduced the number of cytokines from endothelial cells (e.g. MCP-1) and adhesion molecules (P-Selectin and VCAM-1), but not ICAM-1. LXA4, PD1 and RvD1 also improved the uptake of apoptotic thymocytes, which could contribute to the antiatherogenic role of this pathway in mice. This process can also be influenced by diet to influence the severity of atherogenic lesions $[51,52]$. RvE1 is protective of periodontitis. In contrast to LXA4, however, exogenous RvE1 also stimulates bone regeneration in the rabbit model for periodontitis [53]. 
In the murine arthritis model, RvD1 and 17-HDHA reduce pain and tissue damage, shown to be stronger than steroid or pain treatments [54].

Unresolved inflammation, epithelial and microvascular injuries can lead to excessive fibrosis, which impairs organ function. Leukotrienes are professionally effective; and in people with sclerodermal interstitial lung disease, LXA4 is found in the lungs in quantities that do not appear to be enough to counteract the profibrotic factors [55]. In animals, an exogenous aspirin-triggered lipoxin analogue reduces pulmonal fibrosis (triggered by the antibiotic bleomycin [56]) and both LXA4 and benzo-LXA4 reduce fibrosis in kidneys [57, 58]. The exogenous administration of RvE1 and RvD1 protects against renal fibrosis by reducing collagen I and IV, A-SMA and fibronectin. In addition, exogenously administered RvD1 reduces the occurrence of pro-inflammatory mediators formed in response to cigarette smoke and lung toxins [59].

If uncontrolled, chronic inflammation may result in numerous diseases, including obesity and diabetes [60]. In both diseases, peripheral blood markers of inflammation are present in elevated levels after intake of a proinflammatory western type diet [61]. Based on these data Hansen et al. postulated that the use of protectins will be a major step forward in the management of obesity [62]. In a recent study it could be shown that resolvin D1 activates lipoxin A4/formyl peptide receptor 2 (ALX/FPR2), which facilitates cardiac healing. The lack of ALX/FPR2 led to the development of spontaneous obesity and diastolic dysfunction with reduced survival with aging and it amplified leukocyte dysfunction and facilitated profound interorgan non resolving inflammation [63].

All these examples show the ability of SPMs and their precursors to in fluence positively the pro-resolving axis of chronic diseases like the PCOS.

The use of naturally occurring bioactive lipid mediators derived from DHA and EPA, therefore, promises new tools to try to reduce the circulus vicious of visceral adiposities in PCOs women and the corresponding inflammation.

Hence the use of 18-HEPA, 17-HDHA, and 14-HDHA maybe a new approach in this disease.

Funding:

Not applicable. No funding.

Conflict of Interest:

Pedro-Antonio Regidor is employee of Exeltis Healthcare, Anna Müller and Manuela Sailer are emploeyees of Exeltis Germany, Fernando Gonzalez Santos is employee of Solutex Spain, Jose Miguel Rizo is employee of Chemo Spain, Fernando Moreno Egea is CEO of Solutex Spain

Ethical approval: This article does not contain any studies with human participants or animals performed by any of the authors.

Author Contribution

Pedro-Antonio Regidor

Responsible for the concept of resolution of inflammation in PCOS

Anna Müller

Responsible for lietrature research

Manuela Sailer

Responsible for graphic design

Fernando Gonzalez Santos

Responsible for the development of SPMs use in humans and therfeore for basic scientific data 
Jose Miguel Rizo

Responsible for PCOS chapter and data aquisition

Fernando Moreno Egea

Responsible for coordination of the manuscript

Acknowledgment:

Tobias Hummel from die kommunikatöre for the design of the figures

References

1.] Ehrmann DA. Polycystic ovary syndrome. The New England Journal of Medicine. 2005; 352, (12): 1223

- 1236. DOI: 10.1056/NEJMra041536.

2.] Homburg R. Polycystic ovary syndrome - from gynecological curiosity to multisystem endocrinopathy. Human Reproduction. 1996; 11, (1): 29-39. DOI: 10.1093/oxfordjournals.humrep.a019031.

3.] Rotterdam ESHRE/ASRM-Sponsored PCOS Consensus Workshop Group, Revised 2003 consensus on diagnostic criteria and long-term health risks related to polycystic ovary syndrome (PCOS). Human Reproduction. 2004; 19 (1): 41-47. DOI: 10.1093/humrep/deh098.

4.] The Rotterdam ESHRE/ASRM-Sponsored PCOS Consensus Workshop Group, Revised 2003 consensus on diagnostic criteria and long-term health risks related to polycystic ovary syndrome. Fertility and Sterility. 2004; 81 (1): 19-25. DOI: 10.1016/j.fertnstert.2003.10.004.

5.] Ehrmann DA, Cavaghan MK, Barnes RB, Imperial J, Rosenfield RL. Prevalence of impaired glucose tolerance and diabetes in women with polycystic ovary syndrome. Diabetes Care. 1999; 22 (1): 141-146. DOI: $10.2337 /$ diacare.22.1.141.

6.] Genazzani AD, Battaglia C, Malavasi B, Strucchi C, Tortolani F, Gamba O. Metformin administration modulates and restores luteinizing hormone spontaneous episodic secretion and ovarian function in nonobese patients with polycystic ovary syndrome. Fertility and Sterility. 2004; 81 (1): 114-119. DOI: 10.1016/j.fertnstert.2003.05.020.

7.] Ciampelli M, Fulghesu AM, Cucinelli F, Pavone V, Ronsisvalle E, Guido M, Caruso A, Lanzone A. Impact of insulin and body mass index on metabolic and endocrine variables in polycystic ovary syndrome. Metabolism: Clinical and Experimental. 1999; 48 (2): 167-172. DOI: 10.1016/s0026-0495(99)90028-8.

8.] Genazzani AD, Lanzoni C, Ricchieri F, Jasonni VM. Myo-inositol administration positively affects hyperinsulinemia and hormonal parameters in overweight patients with polycystic ovary syndrome. Gynecological Endocrinology.2008; 24 (3): 139-144. DOI: 10.1080/09513590801893232.

9.] Croze ML, Soulage CO. Potential role and therapeutic interests of Myo-inositol in metabolic diseases. Biochimie. 2013; 95 (10): 1811-1827. DOI: 10.1016/j.biochi.2013.05.011.

10.] Diamanti-Kandarakis E, Kandarakis H. The role of genes and environment in the etiology of PCOS. Endocrine. 2006; 30 (1): 19-26. DOI:10.1385/ENDO:30:1:19.

11.] Fauser BC, Diedrich K, Bouchard P, Domínguez F, Matzuk M, Franks S, Hamamah S, Simón C, Devroey P, Ezcurra D, Howles CM. Contemporary genetic technologies and female reproduction. Hum. Reprod. Update. 2011;17 (6): 829-47. DOI:10.1093/humupd/dmr033. PMC 3191938.

12.] Legro RS, Strauss JF. Molecular progress in infertility: polycystic ovary syndrome. Fertil. Steril. 2002; 78 (3): 569-76. DOI:10.1016/S0015-0282(02)03275-2.

13.] Filippou P, Homburg R. Is foetal hyperexposure to androgens a cause of PCOS? Human Reproduction Update. 2017; 23 (4): 421-432. DOI:10.1093/humupd/dmx013. 
14.] Teede H, Deeks A, Moran L. Polycystic ovary syndrome: a complex condition with psychological, reproductive, and metabolic manifestations that impacts on health across the lifespan. BMC Med. 2010; 8 (1): 41. DOI:10.1186/1741-7015-8-41.

15.] Faghfoori Z, Fazelian S, Shadnoush M, Goodarzi R. Nutritional management in women with polycystic ovary syndrome: A review study. Diabetes \& Metabolic Syndrome (Review). 2017; 11 Suppl 1: 429432. DOI:10.1016/j.dsx.2017.03.030.

16.] Bastard J-P, Jardel C, Bruckert E, Blondy P, Capeau J, Laville M, Vidal H, Hainque B. Elevated levels of interleukin 6 are reduced in serum and subcutaneous adipose tissue of obese women after weight loss. Journal of Clinical Endocrinology and Metabolism. 2000; 85 (9): 3338-3342. DOI: 10.1210/jcem.85.9.6839.

17.] O'Rourke RW, Kay T, E. Lyle A. Alterations in peripheral blood lymphocyte cytokine expression in obesity. Clinical and Experimental Immunology. 2006; 146 (1): 39- 46. DOI: 10.1111/j.1365-2249.2006.03186.x.

18.] Festa A, D’Agostino Jr. R, Williams K, Karter AJ, Mayer-Davis EJ, Tracy RP, Haffner SM

The relation of body fat mass and distribution to markers of chronic inflammation. International Journal of Obesity. 2001; 25 (10): 1407-1415. DOI: 10.1038/sj.ijo.0801792.

19.] Diehl MAEA. Nonalcoholic steatosis and steatohepatitis IV. Nonalcoholic fatty liver disease abnormalities in macrophage function and cytokines. American Journal of Physiology. 2002; 282 (1): 1-5, 2002. DOI: 10.1152/ajpgi.00384.2001.

20.] Arkan MC, Hevener AL, Greten FR, Maeda S, Li Z-W, Long JM, Wynshaw-Boris A, Poli G, Olefsky J, Karin M. IKK- $\beta$ links inflammation to obesity-induced insulin resistance. Nature Medicine. 2005; 11(2): 191-198. DOI: $10.1038 / \mathrm{nm} 1185$.

21.] Schonbeck U, Mach F, Sukhova GK, Herman M, Graber P, Kehry MR, Libby P. CD40 ligation induces tissue factor expression in human vascular smooth muscle cells. American Journal of Pathology. 2000; 156(1): 7-14. DOI: 10.1016/S0002-9440(10)64699-8.

22.] Reardon CA, Getz GS. Mouse models of atherosclerosis. Current Opinion in Lipidology. 2001; 12 (2): 167-173. DOI: 10.1097/00041433-200104000-00010.

23.] Michelsen KS, Wong MH, Shah PK, Zhang W, Yano J, Doherty TM, Akira S, Rajavashisth TB, Arditi M. Lack of Toll like receptor 4 or myeloid differentiation factor 88 reduces atherosclerosis and alters plaque phenotype in mice deficient in apolipoprotein E. Proceedings of the National Academy of Sciences of the United States of America. 2004; 101 (29): 10679-10684. DOI: 10.1073/pnas.0403249101.

24.] Cancello R, Tordjman J, Poitou C, Guilhem G, Bouillot JL, Hugol D, Coussieu C, Basdevant A, Hen $\mathrm{AB}$, Bedossa P, Guerre-Millo M, Clément K. Increased infiltration of macrophages in omental adipose tissue is associated with marked hepatic lesions in morbid human obesity. Diabetes. 2006; 55 (6): 1554-1561. DOI: 10.2337/db06-0133.

25.] Licastro F, Candore G, Lio D, Porcellini E, Colonna-Romano G, Franceschi C, Caruso C. Innate immunity and inflammation in ageing: a key for understanding age-related diseases. Immunity and Ageing. 2005; 2: article number 8. DOI: 10.1186/1742-4933-2-8.

26.] Davis JE, Gabler NK, Walker-Daniels J, Spurlock ME. Tlr-4 deficiency selectively protects against obesity induced by diets high in saturated fat. Obesity. 2008; 16 (6): 1248-1255. DOI: 10.1038/oby.2008.210

27.] Schaeffler A, Gross P, Buettner R, et al. Fatty acid-induced induction of Toll-like receptor-4/nuclear factor- $\chi \mathrm{B}$ pathway in adipocytes links nutritional signalling with innate immunity. Immunology. 2009; 126 (2): 233-245. DOI: 10.1111/j.1365-2567.2008.02892.x.

28.] Charriere G, Cousin B, Arnaud E, André M, Bacou F, Penicaud L, Casteilla L. Preadipocyte conversion to macrophage: evidence of plasticity. Journal of Biological Chemistry. 2003; 278 (11): 9850-9855. DOI: 10.1074/jbc.M210811200. 
29.] Bloomgarden ZT. Inflammation and insulin resistance. Diabetes Care 2003; 26 (6): 1922-1926. DOI: 10.2337/diacare.26.6.1922.

30.] Frishman WH. Biologic markers as predictors of cardiovascular disease. American Journal of Medicine. 1998; 104 (6): 18-27. DOI: 10.1016/s0002-9343(98)00184-3.

31.] Orio Jr. F, Palomba S, Cascella T, Sebastiano Di Biase S, Manguso F, Tauchmanovà L, Nardo LG, Labella D, Savastano S, Russo T, Zullo F, Colao A, Lombardi G. The increase of leukocytes as a new putative marker of low-grade chronic inflammation and early cardiovascular risk in polycystic ovary syndrome. Journal of Clinical Endocrinology and Metabolism. 2005; 90 (1): 2-5. DOI: 10.1210/jc.2004-0628.

32.] Flower R J. Prostaglandins, bioassay, and inflammation. Br. J. Pharmacol. 2006; 147: 82-192. DOI: 10.1038/sj.bjp.0706506.

33.] Samuelsson B. Role of basic science in the development of new medicines: examples from the eicosanoid field. J. Biol. Chem. 2012; 287: 10070-10080. DOI: 10.1074/jbc.X112.351437.

34.] Dinarello C A, Simon A, van der Meer J W. Treating inflammation by blocking interleukin-1 in a broad spectrum of diseases. Nature Rev. Drug Discov. 2012; 11: 633-652. DOI: 10.1038/nrd3800.

35.] Serhan CN, Savill J. Resolution of inflammation: the beginning programs the end. Nature Immunol. 2005; 6: 1191-1197. DOI: 10.1038/ni1276.

36.] Maderna P, Godson C. Lipoxins: resolutionary road. Br. J. Pharmacol. 2009; 158, 947-959. DOI: 10.1111/j.1476-5381.2009.00386.x.

37.] Tabas I., Glass C K. Anti-inflammatory therapy in chronic disease: challenges and opportunities. Science. 2013; 339: 166-172. DOI: 10.1126/science.1230720.

38.] Serhan CN, Clish CB, Brannon J, Colgan SP, Chiang N, Gronert K. Novel functional sets of lipidderived mediators with antiinflammatory actions generated from omega-3 fatty acids via cyclooxygenase 2-nonsteroidal antiinflammatory drugs and transcellular processing. J. Exp. Med. 2000;192: 1197-1204. DOI: 10.1084/jem.192.8.1197.

39.] Serhan CN, Hong S, Gronert K, Colgan SP, Devchand PR, Mirick G, Moussignac R-L. Resolvins: a family of bioactive products of omega-3 fatty acid transformation circuits initiated by aspirin treatment that counter pro-inflammation signals. J. Exp. Med. 2002; 196: 1025-1037. DOI: 10.1084/jem.20020760.

40.] Lands WE M. Fish, Omega-3 and Human Health 2nd edn (AOCS Press, 2005) p 34-45.

41.] Serhan C N, Chiang N. Resolution phase lipid mediators of inflammation: agonists of resolution. Curr. Opin. Pharmacol. 2013;13: 632-640. DOI: 10.1016/j.coph.2013.05.012.

42.] Serhan CN, Levy BD. Resolvins in inflammation: emergence of the pro-resolving superfamily of mediators. J Clin Invest. 2018; 128(7): 2657-2669. DOI:org/10.1172/JCI97943.

43.] Serhan CN. Pro-resolving lipid mediators are leads for resolution physiology. Nature 2014; 510: 92-101. DOI:10.1038/nature13479.

44.] Serhan CN. Treating inflammation and infection in the $21^{\text {st }}$ century: new hints from decoding resolution mediators and mechanisms. FASEB J. 2017; 31: 1273-1288. DOI: 10.1096/fj.201601222R.

45.] Lopez-Vicario C. Titos E, Walker ME, Alcaraz-Quiles J, Casulleras M, Duran-Guell M, Flores-Costa R, Perez-Romero N, Forne M, Dalli J, Claria J. Leukocytes from obese individuals exhibit an impaired SPM signature. The FASEB Journal. 2019; 33 (6): 7072-7083. DOI: 10.1096/fj.201802587R.

46.] Ndefo UA, Eaton A, Green MR. Polycystic Ovary Syndrome. A Review of Treatment Options with a Focus on Pharmacological Approaches. P\&T. 2013; 38(6): 336-338, 348, 355. 
47.] Mortada R, Williams T. Metabolic Syndrome: Polycystic Ovary Syndrome. FP Essentials. 2015;435: $30-42$.

48.] Giallauria F, Palomba S, Vigorito C, Tafuri MG, Colao A, Lombardi G, Orio F. Androgens in polycystic ovary syndrome: the role of exercise and diet. Seminars in Reproductive Medicine. 2009; 27 (4): 306-15. DOI:10.1055/s-0029-1225258.

49.] Malawista, S. E., de Boisfleury Chevance, A., van Damme, J. \& Serhan, C. N. Tonic inhibition of chemotaxis in human plasma. Proc. Natl Acad. Sci. USA 105, 17949-17954 (2008)

50.] Serhan, C. N. et al. Reduced inflammation and tissue damage in transgenic rabbits overexpressing 15-lipoxygenase and endogenous anti-inflammatory lipid mediators. J. Immunol. 171, 6856-6865 (2003).

51.] Merched, A. J., Ko, K., Gotlinger, K. H., Serhan, C. N. \& Chan, L. Atherosclerosis: evidence for impairment of resolution of vascular inflammation governed by specific lipid mediators. FASEB J. 22, 35953606 (2008).

52.] Merched, A. J., Serhan, C. N. \& Chan, L. Nutrigenetic disruption of inflammation- resolution homeostasis and atherogenesis. J. Nutrigenet. Nutrigenomics 4, 12-24 (2011).

53.] Hasturk, H. et al. RvE1 protects from local inflammation and osteoclast mediated bone destruction in periodontitis. FASEB J. 20, 401-403 (2006).

54.] Lima-Garcia, J. F. et al. The precursor of resolvin D series and aspirin-triggered resolvin D1 display anti-hyperalgesic properties in adjuvant-induced arthritis in rats. Br. J. Pharmacol. 164, 278-293 (2011).

55.] Kowal-Bielecka, O., Kowal, K., Distler, O. \& Gay, S. Mechanisms of disease: leukotrienes and lipoxins in scleroderma lung disease-insights and potential therapeutic implications. Nature Clin. Pract. Rheumatol. $3,43-51$ (2007).

56.] Martins, V. et al. ATLa, an aspirin-triggered lipoxin A4 synthetic analog, prevents the inflammatory and fibrotic effects of bleomycin-induced pulmonary fibrosis. J. Immunol. 182, 5374-5381 (2009).

57.] Börgeson, E. et al. Lipoxin A(4) and benzo-lipoxin A(4) attenuate experimental renal fibrosis. FASEB J. 25, 2967-2979 (2011).

58.] Qu, X. et al. Resolvins E1 and D1 inhibit interstitial fibrosis in the obstructed kidney via inhibition of local fibroblast proliferation. J. Pathol. 228, 506-519 (2012).

59.] Hsiao, H. M. et al. A novel anti-inflammatory and pro-resolving role for resolvin D1 in acute cigarette smoke-induced lung inflammation. PLoS ONE 8, e58258 (2013).

60.] Serhan, CN. A search for endogenous mechanisms of anti-inflammation uncovers novel chemical mediators: missing links to resolution. Histochem. Cell Biol. 122, 305-321. (2004). DOI: 10.1007/s00418-004-0695-8

61.] Calder, PC. Omega-3 fatty acids and inflammatory processes: from molecules to man. Biochem. Soc. Trans. 45, 1105-1115 (2017). DOI: 10.1042/ BST20160474

62.] Hansen TV, Vik A, Serhan CN. The protectin family of specialized pro-resolving mediators. Potent immunoresolvents enabling innovative approaches to target obesity and diabetes. Frontiers in Pharmacology 9, article 1582 (2019). DOI: org/10.3389/fphar.2018.01582

63.] Tourki B, Kain V, Pullen AB, Norris PC, Patel N, Arora P, Leroy X, Serhan CN, Halade GV. Lack of resolution sensor drives age-related cardiometabolic and cardiorenal defects and impedes inflammationresolution in heart failure. Molecular Metabolism 31138 - 149 (2020). DOI: org/10.1016/j.molmet.2019.10.008

Legends:

Figure 1: The current model of inflammatory processes (modified from Serhan and Levy [42]). 
Figure 2: Novel description of the potential development of inflammatory responses (modified from Serhan [43]).

Figure 3: PEA and DHA metabolic pathways (modified from Serhan [44]).

Figure 4: The pathway of efficacy of the mediators (modified from Serhan [43]).

Figure 5: The use of 17-HDHA and 18-HEPE enhanced the amount of SMP's in fat cells in comparison to cells of healthy weight probands (modified from Lopez Vicario [45]).

Figure 6: Pathway from DHA to the D resolvins (modified from Lopez Vicario [45]).

Figure 1

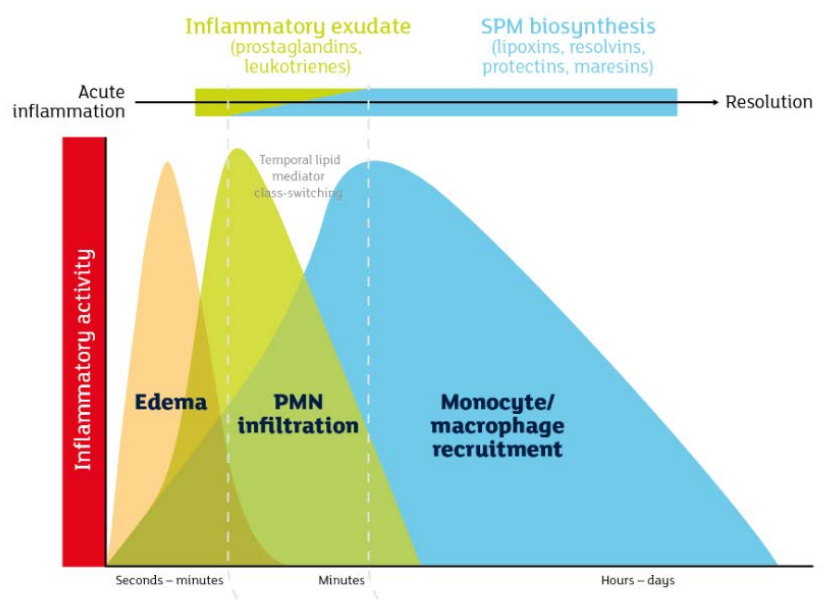




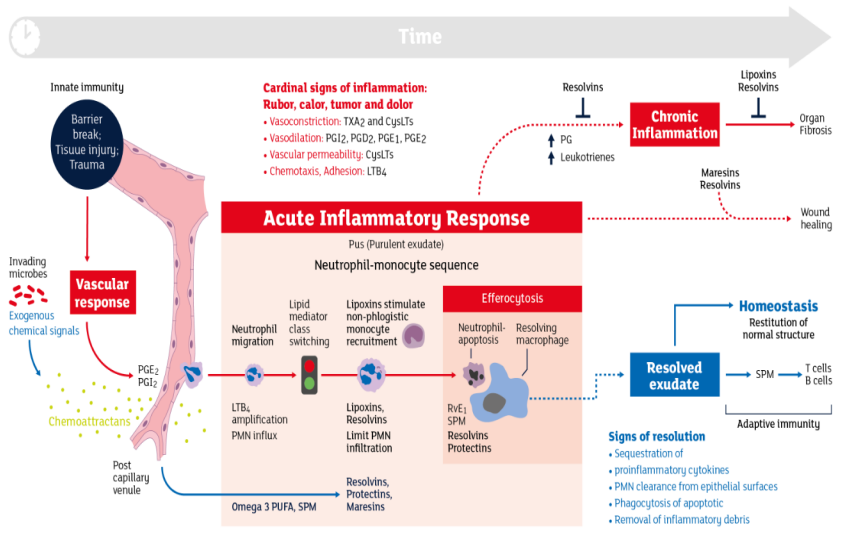




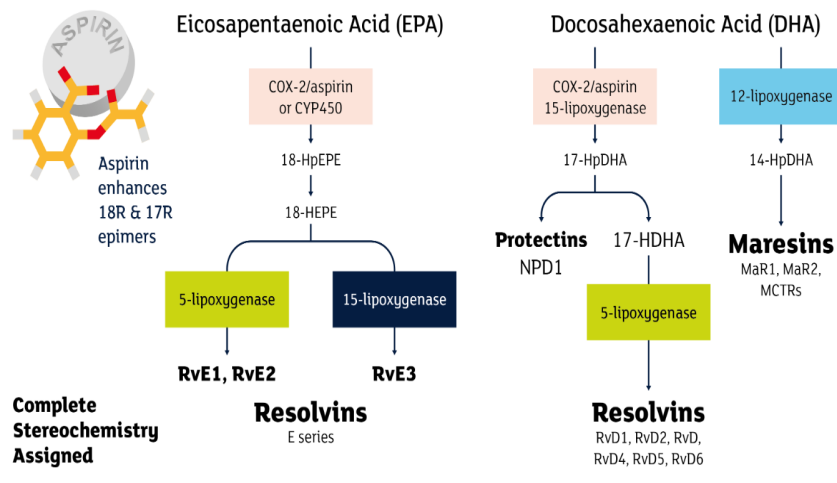


Figure 4

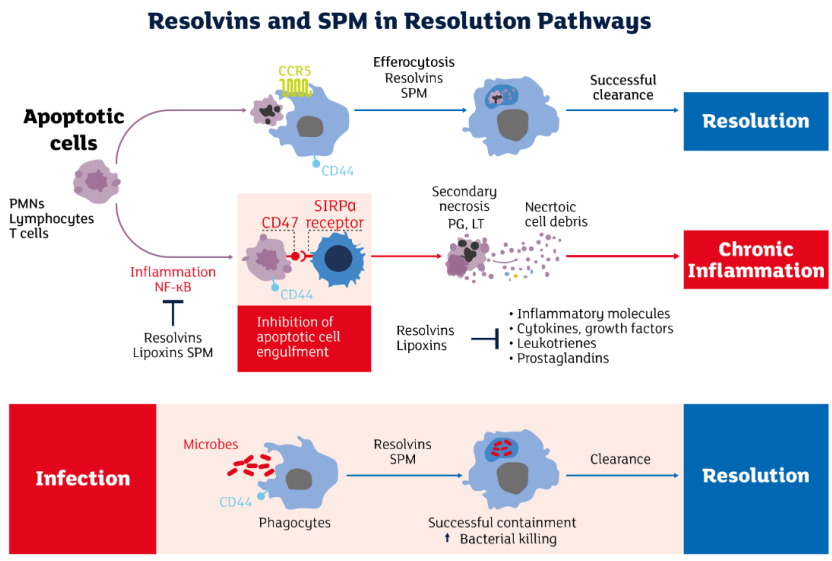


Figure 5
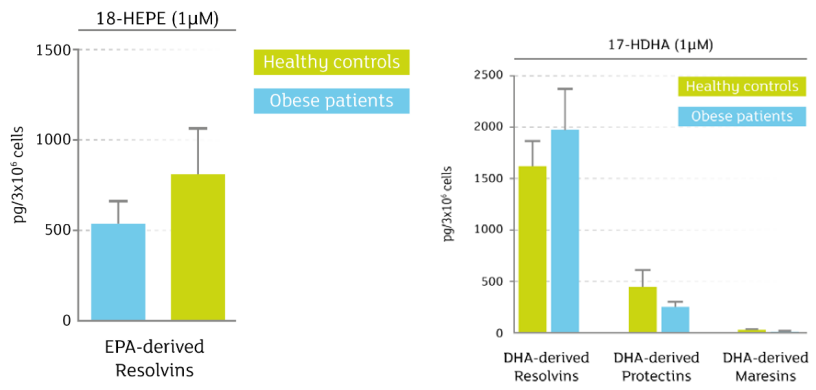
Figure 6

\section{DHA}

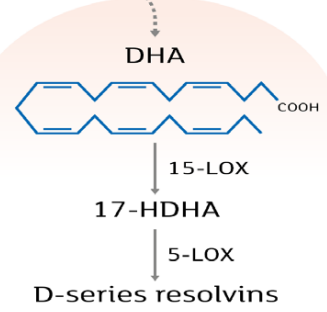

